\title{
A NOVEL REMOVAL METHOD FOR DENSE STRIPES IN REMOTE SENSING IMAGES
}

\author{
Xinxin Liu a , Huanfeng Shen a, e, *, Qiangqiang Yuan ${ }^{\text {b, e }}$, Liangpei Zhang c, e, Qing Cheng ${ }^{\mathrm{d}}$ \\ a School of Resource and Environmental Sciences, Wuhan University, Wuhan 430079, China \\ b School of Geodesy and Geomatics, Wuhan University, Wuhan 430079, China \\ ${ }^{\mathrm{c}}$ LIESMARS, Wuhan University, Wuhan 430079, China \\ d School of Urban Design, Wuhan University, Wuhan 430072, China \\ ${ }^{\text {e }}$ Collaborative Innovation Center for Geospatial Information Technology, Wuhan 430079, China \\ Email: shenhf@whu.edu.cn
}

Youth Forum

KEY WORDS: Destriping, Remote sensing image, Savitzky-Golay (SG) filter, Optimization-based model, Alternating direction method of multipliers (ADMM)

\begin{abstract}
:
In remote sensing images, the common existing stripe noise always severely affects the imaging quality and limits the related subsequent application, especially when it is with high density. To well process the dense striped data and ensure a reliable solution, we construct a statistical property based constraint in our proposed model and use it to control the whole destriping process. The alternating direction method of multipliers (ADMM) is applied in this work to solve and accelerate the model optimization. Experimental results on real data with different kinds of dense stripe noise demonstrate the effectiveness of the proposed method in terms of both qualitative and quantitative perspectives.
\end{abstract}

\section{INTRODUCTION}

Mainly because of inconsistent response and imperfect calibration of each detector (Chen, et al., 2003), striping effects are common phenomena in spaceborne and airborne multidetector remote sensing imaging systems, such as Landsat Thematic Mapper (TM) and the Moderate Resolution Imaging Spectroradiometer (MODIS). These stripe noise can not only greatly reduce the data accuracy but also seriously influence the further applications. Therefore, it is necessary and crucial to remove the stripes and improve the data quality before image interpretation.

As an important pre-processing step, destriping has attracted a lot of research attention in the past decades. Generally speaking, there are three major groups in destriping methods. The first family, including moment matching (Gadallah, et al., 2000; Shen, et al., 2014) and histogram matching (Horn and Woodham, 1979; Wegener, 1990), consist of statistical-based matching approaches and holds the assumption that the distribution properties of the target detector are similar to the reference one. Although with high computational efficiency, the distortion in destriping results is often inevitable when stripes are non-linear or irregular (Shen, et al., 2014). Another class of destriping methods is filteringbased technique (Torres and Infante, 2001; Chen, et al., 2003; Münch, et al., 2009). By well utilizing the transform, the scattered stripe-related information in spatial domain may become concentrated in a particular transform domain, hence the destriping procedure would naturally change into a much simpler way as the truncation of the stripe-related frequency. Unfortunately, the decision of a suited transform or an accurate truncation in this class of approaches is not an easy problem. Until recently, the fast developed optimization-based methods compose the last destriping category (Shen and Zhang, 2009; Bouali and Ladjal, 2011). This kind of technique can make use of striping characteristics or the neighboring pixels' information as prior knowledge to estimate the true image. Since the prior modeling selection is relatively flexible, the optimization-based methods are undoubtedly promising.

Conventionally, according to the common requirement in noise removal area, the maintenance of original healthy information should never be ignored in destriping process. However, in special cases of dense stripes, it is always extremely hard to distinguish the non-striping lines from the whole image. So instead of finding out the uncertain healthy pixels and keeping them intact, a more feasible way is to estimate some local statistical features of latent clean image and then use them to constrain the destriping process.

Inspired from the analysis above, a new destriping method for remote sensing data has been proposed in this work. Unlike the other destriping model, our key idea is to introduce the statistical property constraint in the variational optimization and then use it to ensure a reliable estimation of the true image. To put it more specifically, a rough estimation of statistical along-stripe mean value will be first obtained through a one-dimensional filter, and then constructed in the variational destriping model for a finer solution. An ADMM algorithm (Boyd, et al., 2011) is adopted to solve the minimization problem in this work. MODIS images and Hyperspectral Digital Imagery Collection Experiment (HYDICE) image are both tested in the experiments to validate our proposed approach. The next section of this paper describes the mathematical formulation of the destriping algorithm and is followed by experimental results, conclusion and future work.

\section{DESTRIPING MODEL}

\subsection{Problem Formulation}

In remote sensing images, the striping effect can be considered

* Corresponding author 
as additive noise (Bouali and Ladjal, 2011), so the degradation process can be described as:

$$
\mathbf{f}=\mathbf{u}+\mathbf{s}
$$

where $\mathbf{u}$ is the clean true image, $\mathbf{f}$ is the observed image, and $\mathbf{s}$ denotes different kinds of stripe noise and minor random noise. It's worth noting that the destriping task in this work is to recover $\mathbf{u}$ from the given $\mathbf{f}$ with the presence of $\mathbf{s}$. So in order to solve this typical ill-posed inverse problem, the key becomes to explore and construct appropriate prior terms to suppress the stripe noise but avoid over-smoothing effects.

\subsection{Statistical Property Based Constraint}

Thanks to the special structural property of stripe noise, the along-stripe statistics of one striped data can always reflect the distinct features between striping lines and non-striping lines, and the mean cross-track profile (Gadallah, et al., 2000; Chen, et al., 2003) is just one typical one-dimensional mean statistics among these along-stripe statistics. Since the profiles before and after destriping hold similar tendencies and the corresponding estimation of the latent clean data can be done by filtering the profile of the observations, this statistical feature is very suitable to build the constraint term in our model. Considering the facts that the one-dimensional profile before destriping is consecutive noisy signal which exactly meets the condition of being processed by Savitzky-Golay (SG) filter (Press, et al., 1992), and that the SG filter itself is also a simple but effective method to alleviate the disturbances in the consecutive data, we finally choose the SG filter to smooth the noisy mean profile as:

$$
\hat{\mathbf{M}}_{j}=\frac{\sum_{i=-r}^{r} C_{i} \mathbf{M}_{j+i}}{2 r+1}
$$

where $\mathbf{M}$ and $\hat{\mathbf{M}}$ are the noisy and filtered mean cross-track profile separately, $j$ is the running index of Savitzky-Golay smoothing, and $C_{i}$ denotes the weight for $i$ th mean value in the smoothing window with the radius of $r$.

After obtaining the filtered profile $\hat{\mathbf{M}}$, the next step is to construct the statistical feature in our model. Assuming that a $m \times n \quad \mathbf{u}$ holds $m$ along-stripe lines with $n$ pixels for each line, and the stripes are extending horizontally, the statistical property based constraint can then be defined as:

$$
R_{1}(\mathbf{u})=\|\hat{\mathbf{M}}-\mathbf{u} \times \mathbf{A}\|_{2}^{2}
$$

where A represents a special column vector whose $n$ elements are equal to $1 / n$, and designs to calculate the mean cross-track profile in the desired image. Therefore, the $R_{1}(\mathbf{u})$ is in fact a prior term working to keep the similarity between the recovered feature and the estimated feature, and further functioning to control the whole destriping process for a reliable output.

\subsection{Anisotropic Destriping}

Due to the desirable property as effective preservation of edge information, the TV-based techniques have become a widely used group of models in noise removal area. Although it was first proposed to smooth random noise without particular structure (Radin, et al., 1992), the unsymmetrical version of TV regularization has also been proved to be valid in destriping problem (Bouali and Ladjal, 2011) with the following expression:

$$
R_{2}(\mathbf{u})=\lambda_{1}\left\|\nabla_{x} \mathbf{u}-\nabla_{x} \mathbf{f}\right\|_{1}+\lambda_{2}\left\|\nabla_{y} \mathbf{u}\right\|_{1}
$$

where $\nabla_{x}$ and $\nabla_{y}$ respectively stands for the along-stripe and across-stripe partial differential operator, $\lambda_{1}$ and $\lambda_{2}$ denote the regularization parameters. In $R_{2}(\mathbf{u}),\left\|\nabla_{y} \mathbf{u}\right\|_{1}$ works to smooth the stripe noise by minimizing the across-stripe gradients in $\mathbf{u}$, while $\left\|\nabla_{x} \mathbf{u}-\nabla_{x} \mathbf{f}\right\|_{1}$ aims at maintaining the stripeunaffected gradients from the observed image to the final solution. Owing to this special design, $R_{2}(\mathbf{u})$ can treat the along-stripe and across-stripe gradients in different ways, which exactly meets the unidirectional property of stripe noise.

\subsection{A Joint Destriping Model}

In order to solve a smooth but reliable image from a dense striped data, a joint destriping model combining the statistical feature based constraint $R_{1}(\mathbf{u})$ and the anisotropic regularization $R_{2}(\mathbf{u})$ has been proposed in this work, and expressed as follows:

$$
\hat{\mathbf{u}}=\arg \min E(\mathbf{u})
$$

where

$$
\begin{aligned}
E(\mathbf{u}) & =R_{1}(\mathbf{u})+R_{2}(\mathbf{u}) \\
& =\|\hat{\mathbf{M}}-\mathbf{u} \times \mathbf{A}\|_{2}^{2}+\left(\lambda_{1}\left\|\nabla_{x} \mathbf{u}-\nabla_{x} \mathbf{f}\right\|_{1}+\lambda_{2}\left\|\nabla_{y} \mathbf{u}\right\|_{1}\right)
\end{aligned}
$$

Since the estimation of statistical feature in desired image can be easily attained through noisy profile filtering, and the unsymmetrical TV regularization can well describe the anisotropic property of stripes, the proposed model in (6) is expected to be robust and effective to the dense striped images.

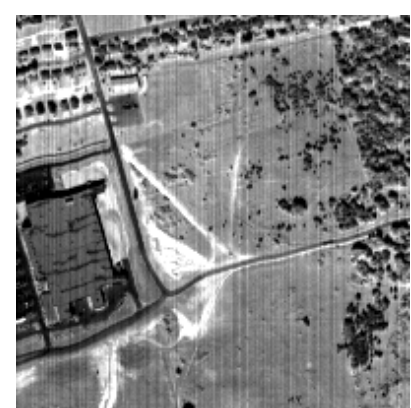

(a)

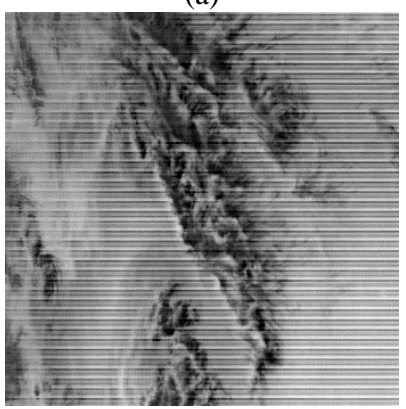

(c)

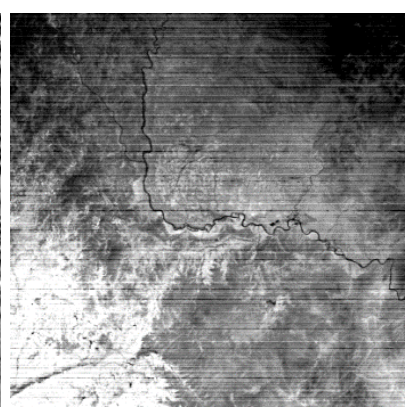

(b)

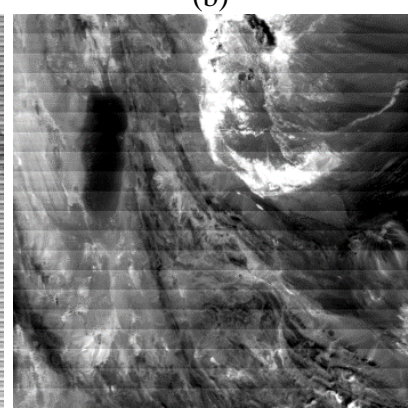

(d)
Fig 1. Experimental images: (a) HYDICE band 103; (b) Terra MODIS band 30; (c) Terra MODIS band 27; and (d) Terra MODIS band 9. 
The difficulties of solving the proposed model (6) mainly lie in the non-differentiability and inseparability $\ell^{1}$-norm terms. To tackle this problem, we adopt ADMM approach (Boyd, et al., 2011) as the ADMM iteration is an efficient tool to solve $\ell^{1}$ minimization and TV-minimization. Some detailed examples of ADMM implementation can be found in the recent related works (Tracey, et al., 2014; Liu, et al., 2016).

\section{EXPERIMENTS AND DISCUSSION}

To verify the effectiveness of the proposed optimization-based destriping method, we choose two types of remote sensing data

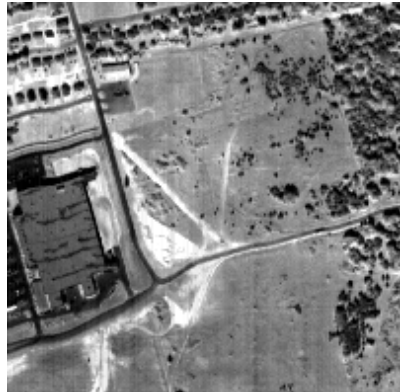

(a)

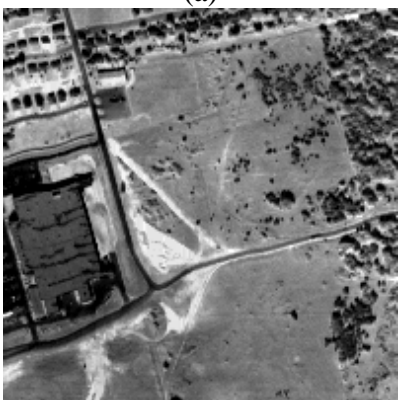

(c)

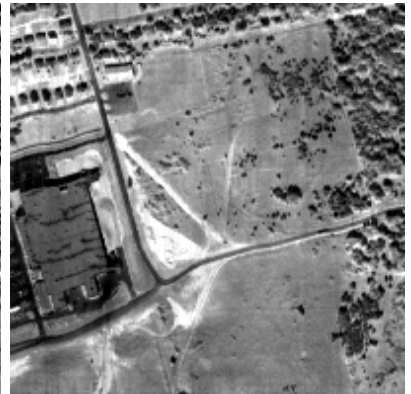

(b)

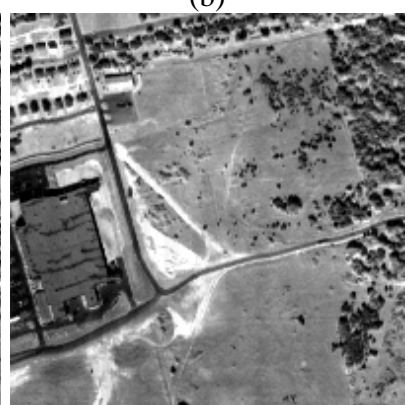

(d)
Fig 2. Destriping results of HYDICE band 103: (a) MM; (b) WAFT; (c) UTV; and (d) the proposed method.

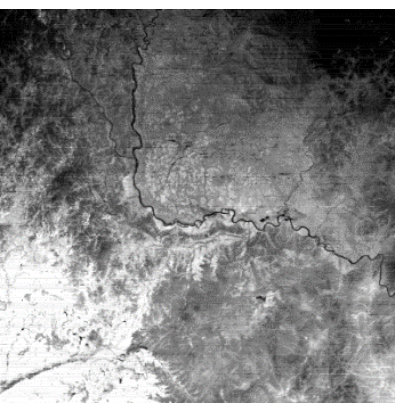

(a)

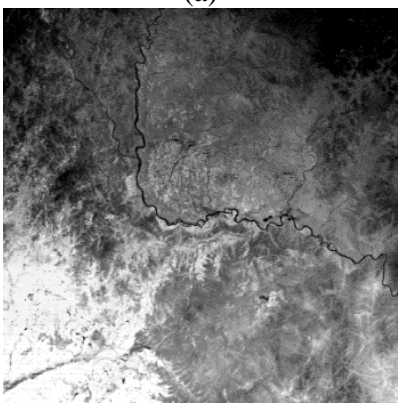

(c)

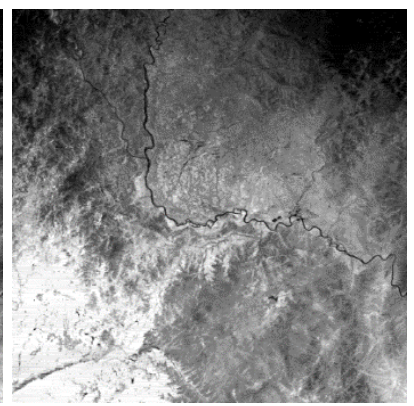

(b)

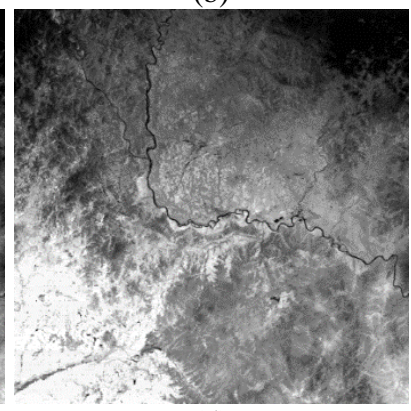

(d)
Fig 3. Destriping results of Terra MODIS band 30: (a) MM; (b) WAFT; (c) UTV; and (d) the proposed method. including the pushbroom-scanning HYDICE data and the whiskbroom-scanning Terra MODIS data. Specifically, one $200 \times 200$ HYDICE subimage and three $400 \times 400$ MODIS subimages with different kinds of dense stripes were extracted from their original versions as experimental images. They are shown in Fig 1. It can be seen that Figs 1(a) and 1(b) are highly contaminated by irregular random stripes, while the stripes in Figs 1(c) and 1(d) are much more regular with apparent periodic properties. In addition, the striping level in Fig 1(c) is the highest among the four experimental images. As the comparisons, the statistical-based moment matching (MM) (Gadallah, et al., 2000),

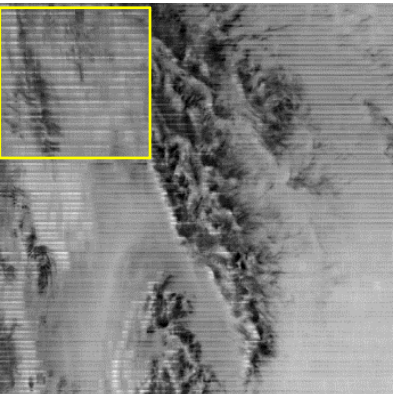

(a)

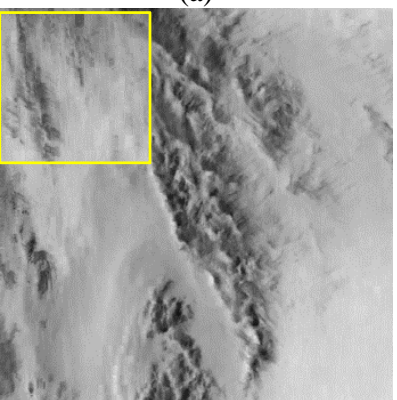

(c)

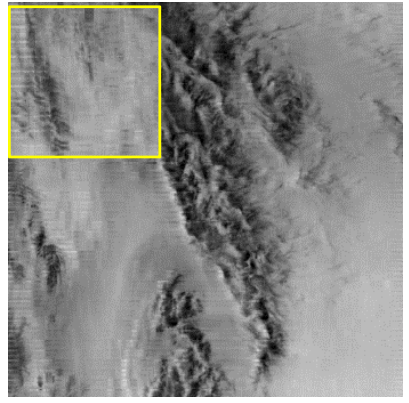

(b)

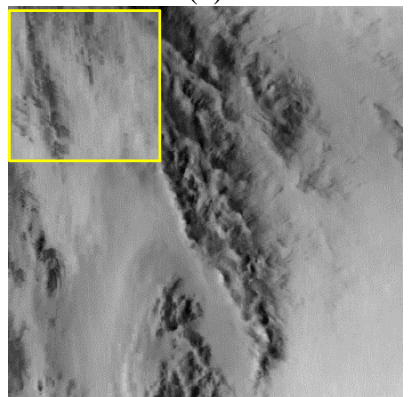

(d)
Fig 4. Destriping results of Terra MODIS band 27: (a) MM; (b) WAFT; (c) UTV; and (d) the proposed method.

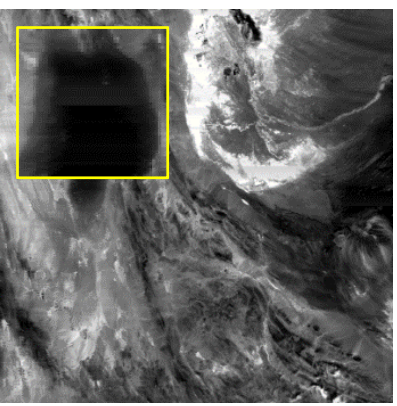

(a)

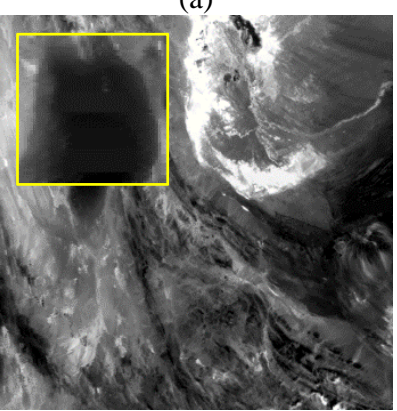

(c)

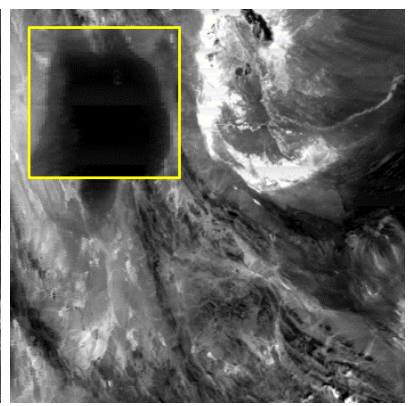

(b)

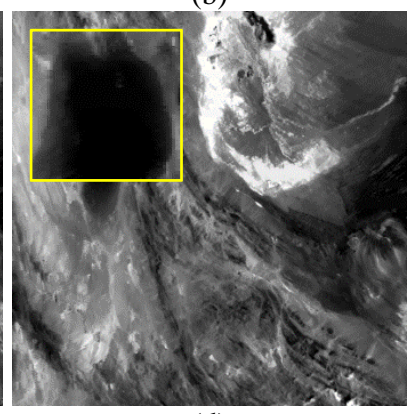

(d)
Fig 5. Destriping results of Terra MODIS band 9: (a) MM; (b) WAFT; (c) UTV; and (d) the proposed method 
the filtering-based combined wavelet-Fourier filtering (WAFT) (Münch, et al., 2009), and the optimization-based unidirectional total variational model (UTV) (Bouali and Ladjal, 2011) were tested in our experiments. For the convenience of quantitative evaluation, test images were normalized between $[0,1]$. Besides, all the experiments were conducted in MATLAB.

Figs 2-5 display the experimental results of four test images. According to the solutions of MM, its performance is unstable. Although the destriping results of the first two test data seem comparable to other methods, the residual artifacts and

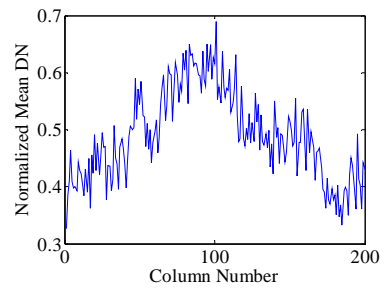

(a)

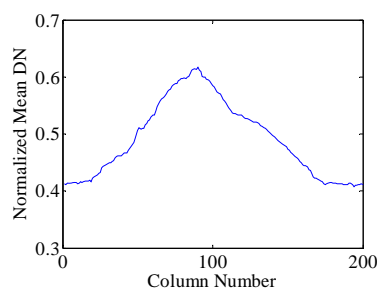

(c)

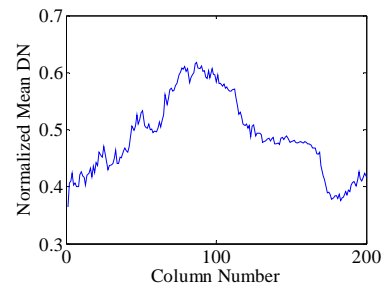

(b)

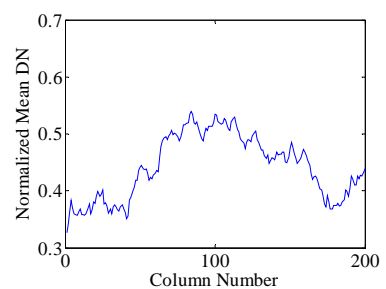

(d)

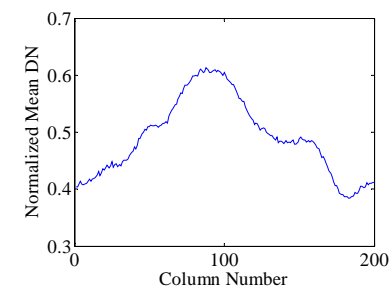

(e)

Fig 6. Mean cross-track profiles of HYDICE band 103:

(a) noisy image; (b) MM; (c) WAFT; (d) UTV; and (e) the proposed method.

Figs 6-7 respectively display the mean cross-track profiles of HYDICE band 103 and Terra MODIS band 27. Although the striping types of these two images are different, good destriping results would output similar profiles which hold same curve trend in the before and after profiles except the sudden disturbances. It is clear that MM can basically keep a right curve trend in its cross-track profiles but cannot well alleviate the disturbances, while UTV just dose the opposite and the over-smoothing effects are significant such as in Fig 7(d). Moreover, the profile of WAFT in Fig 7(c) is satisfying, however the results in Fig 6(c) reveals the unavoidable distortion problem of this method. Unlike the other three techniques, our model both keeps a right curve trend and reduces the corresponding fluctuations, which means our estimations of the true images are good and reliable.

Since all the test images in our experiments are real data with dense stripe noise, only the quantitative measurement related to destriping ability is given in this work. As a non-reference index, the inverse coefficient of variation (ICV) calculated in $10 \times 10$ homogeneous region is used to reflect the level of residual stripe noise (Nichol and Vohora, 2004). Additionally, the higher ICV value means the less remaining noise, and indicates a better destriping result. To decrease the influence of accidental factors, distortions are non-neglectable in Fig 4(a). WAFT can well remove the stripe noise, but the radiance fluctuations may still be obvious in some homogeneous area of the resulting image. The typical examples have been shown in magnified yellow rectangles in Fig 4(b) and Fig 5(b). As for UTV and the proposed method, both of them can provide much more robust destriping results. However without an appropriate constraint to control the processing procedure, UTV is easy to causes over-smoothing phenomenon, e.g., the brightness distortion in Fig 4(c), while our approach is able to conquer this problem due to the design of statistical property based constraint.

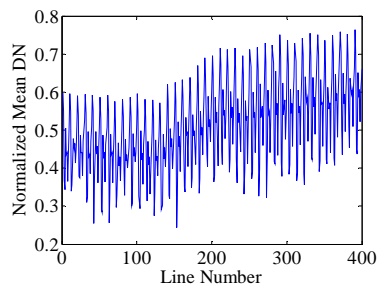

(a)

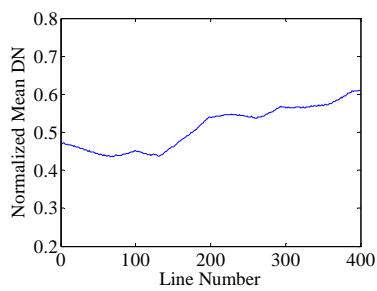

(c)

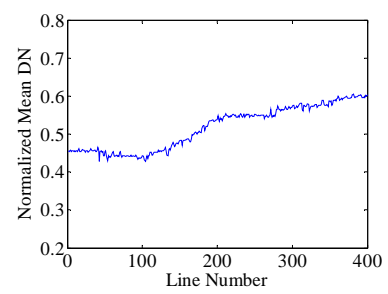

(b)

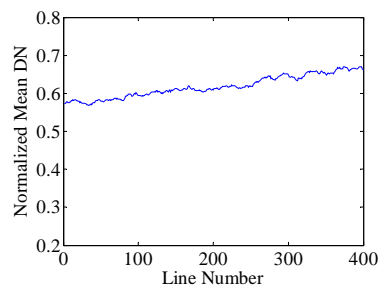

(d)

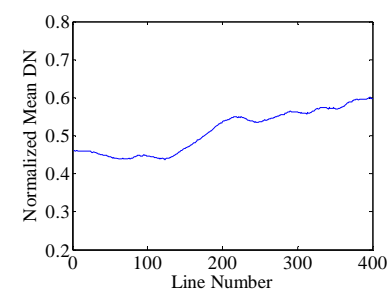

(e)

Fig 7. Mean cross-track profiles of Terra MODIS band 27: (a) noisy image; (b) MM; (c) WAFT; (d) UTV; and (e) the proposed method.

we choose three different samples from every experimental data, and the evaluation is list in Table 1 . According to the results, UTV and the proposed destriping model always obtain the best results, which suggests these two methods have comparative destriping abilities. And this quantitative assessment is in accordance with above visual evaluations.

\begin{tabular}{ccccccc}
\hline \hline Image & Area & Original & MM & WAFT & UTV & Proposed \\
\hline \multirow{2}{*}{ HYDICE } & Sample 1 & 12.36 & 15.03 & 17.90 & 16.24 & $\mathbf{1 8 . 5 3}$ \\
band 103 & Sample 2 & 11.67 & 14.75 & 18.65 & $\mathbf{2 2 . 0 9}$ & 20.08 \\
& Sample 3 & 10.42 & 14.79 & 14.32 & 13.99 & $\mathbf{1 6 . 0 0}$ \\
\hline \multirow{2}{*}{ Terra } & Sample 1 & 7.07 & 12.26 & 13.88 & 11.74 & $\mathbf{1 4 . 3 8}$ \\
band 30 & Sample 2 & 4.65 & 7.95 & 7.65 & 6.63 & $\mathbf{8 . 4 8}$ \\
& Sample 3 & 11.63 & 15.51 & 13.99 & 11.76 & $\mathbf{1 6 . 6 2}$ \\
\hline \multirow{2}{*}{ Terra } & Sample 1 & 4.21 & 17.92 & 22.22 & $\mathbf{2 4 . 5 0}$ & 22.41 \\
band 27 & Sample 2 & 3.63 & 9.08 & 19.82 & 30.88 & $\mathbf{3 2 . 4 8}$ \\
& Sample 3 & 4.50 & 20.96 & 33.15 & $\mathbf{3 8 . 6 5}$ & 34.84 \\
\hline \multirow{2}{*}{ Terra } & Sample 1 & 9.56 & 16.64 & 18.47 & 16.58 & $\mathbf{1 8 . 8 6}$ \\
band 9 9 9 & Sample 2 & 7.36 & 15.38 & 15.25 & 15.84 & $\mathbf{1 7 . 1 8}$ \\
& Sample 3 & 4.80 & 13.40 & 16.45 & $\mathbf{1 7 . 4 0}$ & 16.40 \\
\hline \hline
\end{tabular}

Table 1. Quantitative evaluation results using ICV index 
However, when combining with the visual analyses and considering the ability of maintaining the similar statistical property from the original noisy data, i.e., the overall assessment for the reliability of the solved images, our method can doubtlessly outperform UTV method.

\section{CONCLUSION}

In this work, we have proposed a new destriping method for dense striped remote sensing images. The proposed model can not only effectively remove the heavy stripes but also control the whole destriping process to ensure a reliable estimation. Several real data sets were tested in our experiments. Both the qualitative and quantitative assessments proved that the proposed model can produce better destriping results than the other three techniques including MM, WAFT, and UTV. Besides, the proposed approach is quite robust to different types of dense stripes according to the test. In the future, we will try to modify our model to speed up the calculation for the large scale data processing.

\section{ACKNOWLEDGEMENTS}

This work was supported by the National High Technology Research and Development Program of China (863 Program) under Grant No. 2013AA12A301.

\section{REFERENCES}

Bouali, M., and Ladjal, S., 2011. Toward optimal destriping of MODIS data using a unidirectional variational model. IEEE Trans. Geosci. Remote Sens., 49(8), pp. 2924-2935.

Boyd, S., Parikh, N., Chu, E., Peleato, B., and Eckstein, J., 2011. Distributed optimization and statistical learning via the alternating direction method and multipliers, Found. Trends Mach. Learn., 3(1), pp 1-122.

Chen, J., Shao, Y., Guo, H., Wang, W., and Zhu, B., 2003. Destriping CMODIS data by power filtering. IEEE Trans. Geosci. Remote Sens., 41(9), pp. 2119-2124.

Gadallah, F. L., Csillag, F., and Smith, E. J. M., 2000. Destriping multisensor imagery with moment matching. Int. J. Remote Sens., 21(12), pp. 2505-2511.
Horn, B. K., and Woodham, R. J., 1979. Destriping Landsat MSS images by histogram modification. Comput. Graph. Image Process., 10(1), pp. 69-83.

Liu, X., Lu, X., Shen, H., Yuan, Q. , Jiao, Y., and Zhang, L., 2016. Stripe Noise Separation and Removal in Remote Sensing Images by Consideration of the Global Sparsity and Local Variational Properties. IEEE Trans. Geosci. Remote Sens., DOI: 10.1109/TGRS.2015.2510418.

Münch, B., Trtik, P., Marone, F., and Stampanoni, M., 2009. Stripe and ring artifact removal with combined wavelet-Fourier filtering. Opt. Express., 17(10), pp. 8567-8591.

Nichol, J. E. and Vohora, V., 2004. Noise over water surfaces in Landsat TM images. Int. J. Remote Sens., 25(11), pp. 2087-2094.

Press, W. H., Teukolsky, S. A., Vetterling, W. T., and Flannery, B. P., 1992. Numerical recipes in FORTRAN: the art of scientific computing. Cambridge: Univ. press., London, pp. 963.

Radin, L., Osher, S., and Fatemi, E., 1992. Non-linear total variation noise removal algorithm. Physica D., 60(1), pp. 259268.

Shen, H., Jiang, W., Zhang, H., and Zhang, L., 2014. A piecewise approach to removing the nonlinear and irregular stripes in MODIS data. Int. J. Remote Sens., 35(1), pp. 44-53.

Shen, H., and Zhang, L., 2009. A MAP-based algorithm for destriping and inpainting of remotely sensed images. IEEE Trans. Geosci. Remote Sens., 47(5), pp. 1492-1502.

Torres, J., and Infante, S. O., 2001. Wavelet analysis for the elimination of striping noise in satellite images. Opt. Eng., 40(7), pp. 1309-1314.

Tracey, B. H., Miller, E. L., Wu, Y., Natarajan, P., and Noonan, J. P., 2014. A constrained optimization approach to combining multiple non-local means denoising estimates. Signal Process., 103(10), pp. 60-68.

Wegener, M., 1990. Destriping multiple sensor imagery by improved histogram matching. Int. J. Remote Sens., 11(5), pp. 859-875. 